\title{
UMA CONTRIBUIÇÃO AO DESENVOLVIMENTO E IMPLEMENTAÇÃO DE ROTINAS DE CONTROLE PREDITIVO EM SCILAB
}

\author{
T. C. S. P. BATISTA ${ }^{1}$ e T. V. COSTA ${ }^{1}$ \\ ${ }^{1}$ Universidade Federal de Itajubá, Instituto de Recursos Naturais, Engenharia Química \\ E-mail para contato: thiagocosta@unifei.edu.br
}

\begin{abstract}
RESUMO - As indústrias, cada vez mais, têm empregado técnicas de controle avançado em sua produção em busca de maior produtividade e competitividade. Uma das técnicas mais utilizadas é o Controle Preditivo Baseado em Modelo (MPC), algoritmo que faz uso do modelo da planta para a obtenção de uma sequência de ações de controle, por meio de uma rotina de otimização. Assim, o presente trabalho teve como objetivos o estudo e a aplicação experimental de uma variação do MPC, o Controle Preditivo Generalizado (GPC). Para isso, o algoritmo GPC foi implementado no software livre Scilab, através de diagrama de blocos considerando o módulo Xcos. Para fins de validação, o algoritmo de controle implementado foi aplicado a um protótipo de controle experimental. Os resultados obtidos comprovaram a eficiência do algoritmo GPC em controlar processos simples e complexos, com ou sem restrições. Verificou-se ainda a elevada capacidade do software livre Scilab na análise experimental de estruturas de controle avançado em problemas da Engenharia Química.
\end{abstract}

\section{INTRODUÇÃO}

O controle e a automação de processos apresentam extrema importância na prática industrial, pois possibilitam a geração de produtos com características uniformes, de alta qualidade e capazes de atender as especificações dos clientes e as legislações ambientais. Além disso, o controle garante a segurança dos processos e permite que o sistema trabalhe nas condições de operação desejadas.

Dentre as técnicas de controle avançado adotadas no meio industrial, uma das mais utilizadas, principalmente na indústria química, e uma das poucas capazes de controlar eficientemente sistemas multivariáveis, com restrições e não linearidades é o controle preditivo baseado em modelo (MPC). O MPC corresponde a uma classe de métodos de controle que utilizam o modelo do processo para prever o comportamento futuro da planta e, assim, implementar uma rotina de otimização para o cálculo de uma sequência ótima de ações de controle.

Uma das ferramentas computacionais que podem suportar a implementação de algoritmos de controle preditivo é o Scilab, um software livre e em código aberto (opensource) para computação numérica, que pode ser executado em diversas plataformas 
(Patil et al., 2012). Embora seu uso em aplicações experimentais ainda seja modesto comparado a softwares proprietários, observa-se um aumento gradual em sua utilização no desenvolvimento e análise de estruturas de controle. Como exemplos, pode-se citar seu uso no teste de estruturas de controle avançado utilizando interface com linguagem C\# e comunicação Modbus (Sencio et al., 2013) e também em sistemas mecatrônicos que utilizam tecnologia baseada no protocolo de comunicação digital CAN (Controller Network Area) (Bucher e Balemi, 2010).

Neste contexto, o presente trabalho tem como objetivos o estudo e a avaliação da estratégia GPC (algoritmo pertencente à classe dos controladores MPC), a implementação da técnica em Scilab a partir de diagrama de blocos no módulo Xcos e a aplicação do GPC a um sistema experimental, para fins de validação do algoritmo implementado. Busca-se também verificar a eficiência do método GPC no controle de processos sob a imposição de restrições. Procura-se, deste modo, apresentar uma contribuição ao desenvolvimento e implementação de rotinas de controle preditivo em Scilab e disseminar o uso do software livre na análise experimental de estruturas de controle avançado em problemas da Engenharia Química.

\section{MATERIAIS E MÉTODOS}

\subsection{Fundamentação teórica}

A Equação 1 representa a função objetivo do problema de otimização do controlador GPC. Neste trabalho, optou-se por utilizar um modelo em equações de diferença com estrutura ARX (autoregressive model with exogenous input) de acordo com a restrição de igualdade apresentada na Equação 2. Além disso, outros tipos de restrição a que o problema de otimização pode estar sujeito são restrições de desigualdade sobre a velocidade da variável de controle $(\Delta u)$ e sobre a amplitude da variável de controle $(u)$.

$$
\begin{array}{cc}
\underset{\Delta U}{\operatorname{minimizar} J}=\left(\hat{Y}-Y_{\text {ref }}\right)^{T} \bar{Q}_{y}\left(\hat{Y}-Y_{\text {ref }}\right)+\Delta U^{T} \bar{Q}_{u} \Delta U \\
\text { sujeito à: } & \hat{Y}=G \Delta u+H^{\prime} Y_{\text {passado }}+H^{\prime \prime} \Delta u_{\text {passado }} \\
& u_{\text {min }} \leq u(k-1) \leq u_{\max } \\
& \Delta u_{\text {min }} \leq \Delta u(k-1) \leq \Delta u_{\max }
\end{array}
$$

Sendo $\hat{Y} \in \mathbb{R}^{H p}$ a saída predita do modelo do processo no horizonte de predição $H p ; Y_{\text {ref }} \in$ $\mathbb{R}^{H p}$ o vetor da trajetória de referência; $\Delta U \in \mathbb{R}^{H c}$ o vetor de saídas no horizonte de controle $H c$ e $\bar{Q}_{y} \in \mathbb{R}^{H p \times H p}$ e $\bar{Q}_{u} \in \mathbb{R}^{H c \times H c}$ matrizes bloco diagonais baseadas nas matrizes de pesos para as saídas (variáveis manipuladas) e entradas (variáveis controladas), respectivamente. Observa-se ainda que $\mathrm{G}, H^{\prime}, H^{\prime \prime}, Y_{\text {passado }}, \Delta u_{\text {passado, }}$, são matrizes e vetores obtidos pela recursão do modelo de predição ARX, conforme detalhado em Rossiter (2003).

Para os processos em que é necessário levar em conta as restrições, o problema de controle não possui uma solução analítica, devendo ser resolvido, a cada intervalo de amostragem, através de métodos de programação quadrática ( $\mathrm{QP}$, quadratic programming). Métodos numéricos eficientes na solução dos problemas QP estão disponíveis em rotinas 
computacionais, e consideram as matrizes características do problema de otimização e suas restrições em seus cálculos. Neste trabalho, utilizou-se a rotina qpsolve do software Scilab.

\subsection{Protótipo experimental de controle}

Para fins de validação da estratégia de controle estudada no presente trabalho, foi utilizado o protótipo instrumentado de controle de processos apresentado na Figura 1 a seguir.

Figura 1- Protótipo experimental de controle de processos Edibon UCP-UB

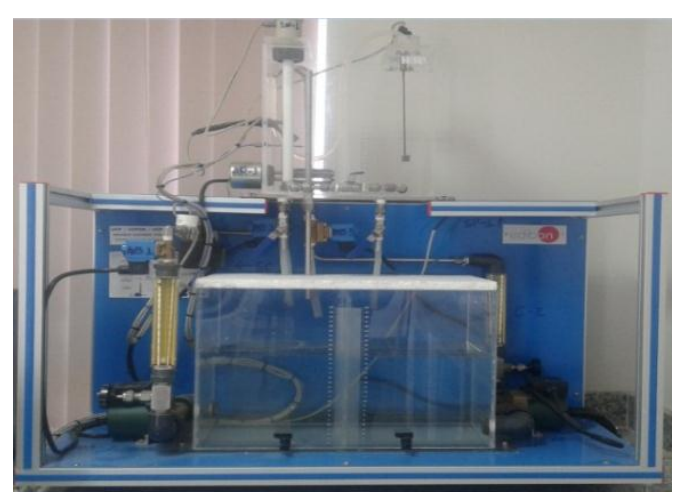

(a) Protótipo experimental

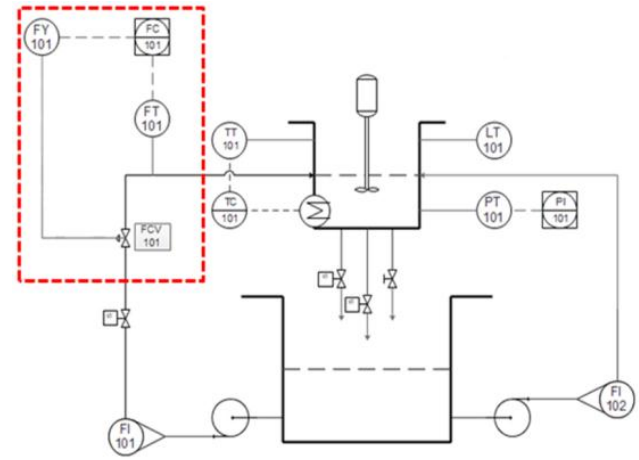

(b) Diagrama de instrumentação

O sistema consiste em um tanque de abastecimento conectado a duas bombas centrífugas que alimentam as correntes de entrada para o tanque superior. Existem três possíveis malhas de controle: nível, vazão e temperatura. Verificou-se que a malha de vazão para este processo representava maiores desafios, como dados sujeitos a ruído de instrumentação e a exigência de uma alta frequência de amostragem para coleta de dados. Por essa razão e devido ao mau funcionamento do atuador referente à malha de temperatura, impossibilitar um estudo em cenário multivariável adequado, escolheu-se aplicar o controle GPC à vazão da corrente que alimenta o tanque superior, conforme indicado na Figura 1 (b). O controle da vazão foi realizado utilizando como variável manipulada a porcentagem de abertura da válvula eletrônica de controle (FCV-101).

O protótipo experimental possui originalmente um software proprietário que não contempla o uso de estruturas de controle avançado. Deste modo, houve a necessidade de implementar rotinas em Scilab para leitura de dados e atuação na unidade de controle por meio de uma interface de comunicação, permitindo que o algoritmo de otimização com restrições fosse utilizado no cálculo das ações de controle. A comunicação do software Scilab com a planta do processo foi realizada através de uma placa de aquisição National Instruments (módulo de aquisição NI-6220). As rotinas computacionais de interface entre o processo e o computador usado na comunicação e aquisição de dados foram implementadas a partir do módulo denominado NI-DAQmx, que consiste num conjunto de funções disponíveis em uma dll (Dynamic Link Library) disponibilizadas pelo desenvolvedor da placa de comunicação. A interface entre tais rotinas e o Scilab foi realizada com base em um toolbox NIDAQ (versão 0.3.1) de rotinas em linguagem $C$ que permite a leitura e escrita das variáveis do processo. 


\section{RESULTADOS E DISCUSSÃO}

\subsection{Identificação e validação do modelo}

A obtenção dos dados para a identificação do modelo do processo foi realizada em malha aberta por meio de alterações na variável manipulada aplicando-se um sinal PRBS (Pseudo-Random Binary Sequence) durante 50 segundos, conforme a Figura 2 (b). O tempo de amostragem empregado no sistema foi de 0,5 segundos. Foram realizados dois testes independentes, gerando assim dois conjuntos de dados distintos. $\mathrm{O}$ primeiro conjunto foi usado para identificar o modelo dinâmico do sistema e o segundo, para fins de validação. A partir dos dados do primeiro conjunto foram estimados os parâmetros de um modelo ARX de acordo com a Equação (3), sendo $\mathrm{Y}(\mathrm{z})$ a variável de saída correspondente à vazão da corrente de alimentação (L/min) e $\mathrm{U}(\mathrm{z})$ a variável de entrada correspondente à porcentagem de abertura de válvula (\%). A comparação entre os dados do processo utilizados para validação e os dados do modelo ARX pode ser observada na Figura 2 (a).

$$
Y(z)=\frac{0,0220 z^{-1}+0,0028 z^{-2}-0,0044 z^{-3}-0,0027 z^{-4}-0,0079 z^{-5}-0,0089 z^{-6}}{1-0,011 z^{-1}-0,215 z^{-2}-0,262 z^{-3}-0,148 z^{-4}-0,330 z^{-5}-0,021 z^{-6}} U(z)
$$

A análise da capacidade de generalização do modelo foi realizada a partir das funções auto-correlação (FAC) e correlação cruzada (FCC) conforme apresentada nas Figuras 2 (c) e 2 (d). Como as funções não ultrapassam o intervalo de confiança, pode-se afirmar que o ruído do processo não está autocorrelacionado e não há correlação entre o ruído e o sinal de entrada PRBS aplicado à planta. Assim, pode-se afirmar que o modelo identificado possui bom desempenho em descrever a dinâmica do processo e tem capacidade de generalização, podendo ser utilizado de forma eficiente para um conjunto de dados diferentes.

Figura 2 - Validação e análise do modelo identificado

(a)

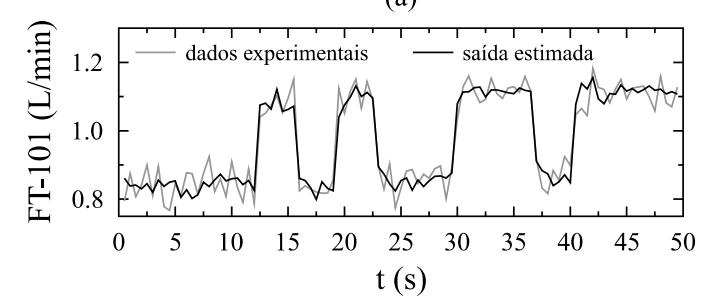

(c)

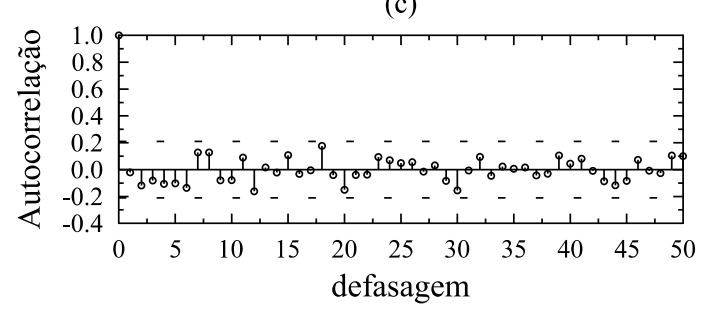

(b)

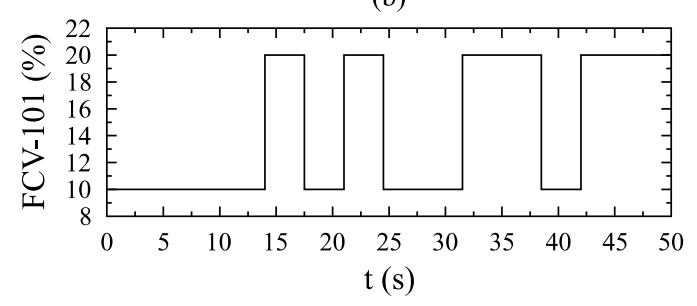

(d)

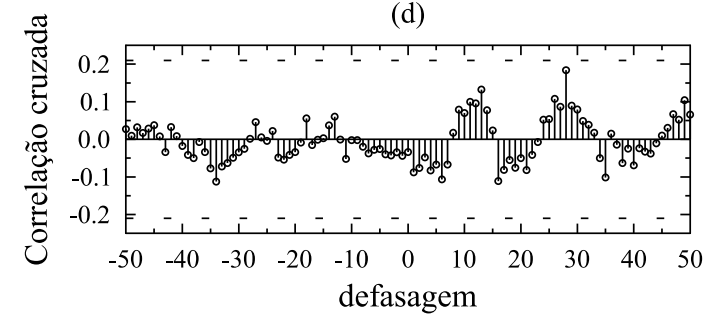

\subsection{Projeto e sintonia do controlador}

O controlador GPC foi desenvolvido e implementado em Scilab a partir de diagrama de 
blocos no módulo Xcos (Figura 3), de acordo com as seguintes etapas: (i) leitura e aquisição de dados do processo; (ii) predição da saída utilizando o modelo identificado e os dados obtidos; (iii) cálculo do desvio da saída em relação à referência; (iv) cálculo da sequência de ações de controle a partir da solução do problema de otimização; (v) implementação do primeiro elemento da sequência de controle.

Figura 3 - Diagrama em Xcos com controlador GPC aplicado ao controle de vazão do protótipo experimental de controle de processos.

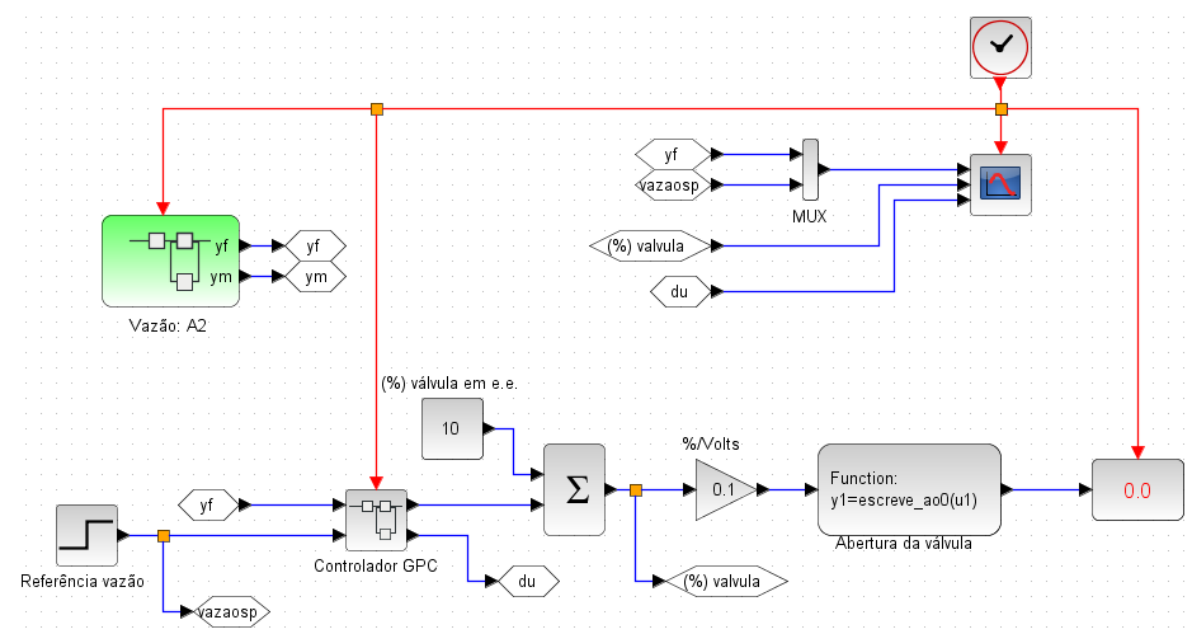

A sintonia do controlador foi realizada de acordo com Moudgalya (2007) e os parâmetros obtidos foram: $H_{p}=30, H_{c}=10, q_{y}=10$ e $q_{u}=0,01$. Foram ainda aplicadas restrições sobre o incremento da variável de controle $(\Delta u)$ e sobre a ação de controle $(u)$, sendo: $-1 \% \leq \Delta u \leq 1 \%$ e $0 \% \leq u \leq 45 \%$.

Para demonstrar a eficácia do algoritmo de controle, simulação e testes experimentais em malha fechada foram realizados. Uma sequência de alterações no setpoint de vazão $(1,1$ $\mathrm{L} / \mathrm{min}-1,5 \mathrm{~L} / \mathrm{min}-1,8 \mathrm{~L} / \mathrm{min}-1,3 \mathrm{~L} / \mathrm{min}$ ) foi aplicada e as ações do sistema de controle observadas conforme apresentado na Figura 4.

Figura 4 - Simulação e experimento em malha fechada para o controle de vazão.

(a)
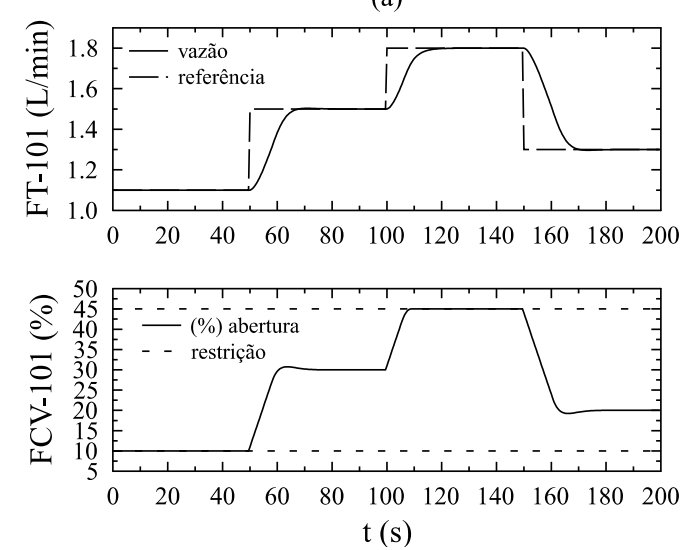

(b)
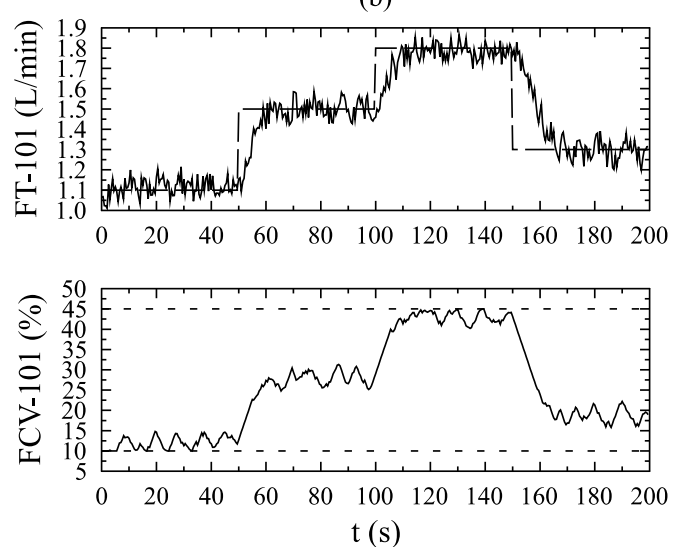
Primeiramente, aplicou-se o algoritmo de controle GPC com a sintonia proposta em um modelo simplificado do processo, identificado a partir de uma perturbação degrau e aproximado para um sistema de primeira ordem com tempo morto. Os resultados da simulação em malha fechada são apresentados na Figura 4 (a), na qual observou-se um bom desempenho do sistema de controle. Em seguida, o mesmo algoritmo de controle foi aplicado ao processo experimental e as respostas do sistema podem ser vistas na Figura 4 (b).

A partir da comparação dos resultados da simulação e dos resultados experimentais, é possível notar que o comportamento do sistema real se aproximou muito da simulação realizada. Além disso, a análise do comportamento em malha fechada permite verificar que, como esperado, o controlador GPC se mostrou apto em alcançar os objetivos de controle estabelecidos, mantendo a variável de saída (vazão) na referência desejada, sem infringir os limites impostos à variável de entrada (restrições sobre a ação de controle).

\section{CONCLUSÕES}

Este trabalho consistiu no estudo, avaliação e implementação da estratégia de controle preditivo generalizado (GPC) no software livre Scilab por meio de diagramas de blocos do módulo Xcos, com posterior aplicação a um sistema experimental de controle de vazão. Com base no estudo de caso foi possível apresentar o uso do software livre na análise de estruturas de controle avançado e demonstrar a capacidade que o Scilab apresenta em auxiliar o desenvolvimento e análise de controladores preditivos. O controlador GPC implementado apresentou bom desempenho mesmo em casos com restrições, sendo capaz de atingir os objetivos de controle determinados, sem infringir as restrições estabelecidas. É importante ressaltar ainda que o algoritmo de controle foi executado no intervalo de amostragem sem que problemas no cálculo das ações de controle tenham sido identificados. Dessa forma, pôde-se comprovar a eficiência do controlador preditivo baseado em modelo no controle de problemas de Engenharia Química a partir de uma ferramenta livre e em código aberto.

\section{REFERÊNCIAS}

BUCHER, R.; BALEMI, S.; MEZA, C.. Open Source Applications for Rapid Control Prototyping in Education Laboratories , XVIII Congresso Brasileiro de Automática, Bonito, p. 12-16, 2010.

MOUDGALYA, K., Digital Control. England, John Wiley \& Sons, 2007.

PATIL, J., DUBEY, B., MOUDGALYA, K. , PETER, R., GNURadio, Scilab, Xcos and COMEDI for Data Acquisition and Control: An Open Source Alternative to LabVIEW, 8th IFAC Symposium on Advanced Control of Chemical Processes, p. 626-631, 2012.

ROSSITER, J., Model-based predictive control: a practical approach. CRC press, 2003.

SENCIO, R., COSTA, T., SILVA, F., Desenvolvimento de uma ferramenta para aplicação experimental e avaliação de estruturas de controle avançado, X Congresso Brasileiro de Engenharia Química Iniciação Científica, 2013. 